\title{
Pulmonary Blastoma
}

National Cancer Institute

\section{Source}

National Cancer Institute. Pulmonary Blastoma. NCI Thesaurus. Code C3732.

A biphasic tumor that consists of fetal adenocarcinoma (typically low-grade) and primitive mesenchymal stroma. Foci of specific mesenchymal differentiation (osteosarcoma, chondrosarcoma, or rhabdomyosarcoma) may also be present, but are not required for the diagnosis. Most patients are smokers. The prognosis is very poor. (WHO 2015) 